\title{
Flow Regime Classification Using Artificial Neural Network Trained on Electrical Capacitance Tomography Sensor Data
}

\author{
Khursiah Zainal-Mokhtar \\ School of Electrical and Electronic, Engineering Campus University Science Malaysia \\ 14300 Nibong Tebal, Penang, Malaysia \\ Tel: 006-0195653288 E-mail: ven_conmigo1010@yahoo.com \\ Junita Mohamad-Saleh \\ School of Electrical and Electronic, Engineering Campus University Science Malaysia \\ 14300 Nibong Tebal, Penang, Malaysia \\ Tel: 006-0194732732 E-mail:.jms@eng.usm.my \\ Hafizah Talib \\ School of Electrical and Electronic, Engineering Campus University Science Malaysia \\ 14300 Nibong Tebal, Penang, Malaysia \\ Tel: 006-0134181851 E-mail: smiley_ahaks@yahoo.com \\ Najwan Osman-Ali \\ School of Electrical and Electronic, Engineering Campus University Science Malaysia \\ 14300 Nibong Tebal, Penang, Malaysia \\ Tel: 006-0195435865 E-mail: ee73620@eng.usm.my
}

The research is fully funded by Ministry of Science, Technology and Innovation (MOSTI), Malaysia under eScienceFund grant.

\begin{abstract}
The main goal of the presented work is to analyse the performance of the Multi-Layer Perceptron (MLP) neural network for flow regime classification based on sets of simulated Electrical Capacitance Tomography (ECT) data. Normalised ECT data have been used to separately train several MLPs employing various commonly used back-propagation learning algorithms, namely the Levenberg-Marquardt (LM), Quasi-Newton (QN) and Resilient-Backpropagation (RP), to classify the gas-oil flow regimes. The performances of the MLPs have been analysed based on their correct classification percentage (CCP). The results demonstrate the feasibility of using MLP, and the superiority of LM algorithm for flow regime classification based on ECT data.
\end{abstract}

Keywords: Multi-Layer Perceptron, Back-propagation algorithm, Electrical Capacitance Tomography, flow regime, Classification

\section{Introduction}

Knowledge of flow regimes is essential particularly for investigation involving multi-component flows such as in oil production. Many types of flow regime could interchangeably form along the pipeline during oil transportation. Since pipelines are normally concealed, a special technique is required in order to obtain information regarding flow regimes form in a pipeline.

Tomographic method was first applied in medical imaging in 1970s when hospital doctors used Computerised Tomography (CT) machine to visualise internal organs of a human body. Nowadays, there are various other tomography systems and ECT is one of the commonly used sensing methods in industries (Beck and Williams, 1996). ECT sensor is non-invasive in nature since it does not need to be inserted inside the pipeline, in which case, may intrude the material permittivity distribution. Moreover, it is non-hazardous since it involves electrical lines unlike CT machines which deal with radiation. Figure 1 illustrates a basic ECT system consisting of the sensors, data acquisition system and a computer system (Gamio et al., 2005). Using this technique, multiple electrodes are mounted equally around the periphery of an 
insulating pipe vessel. These electrodes are sensitive to the dielectric constants of materials and the capacitance variations occur between combinations of electrodes. Measurements between electrode pairs give the capacitance values.

Conventionally, the capacitance values were used to determine the permittivity distribution with the aid of an image reconstruction algorithm. Then, the type of flow regime can be determined from the reconstructed image. However, image reconstruction method is too time-consuming particularly, when online process control is of main concern (Mohamad-Saleh and Hoyle, 2002). In addition, reconstructed images usually have low fidelity and thus, flow process parameter estimations based on the images are typically imprecise. Although rather accurate reconstructed images can be obtained by employing artificial neural network (Marashdeh et al., 2006) as the reconstruction mechanism, the reconstruction time is still a problem (Mohamad-Saleh and Hoyle, 2002).

Historically, the development of ANN technology was motivated by human brain's ability to solve very complex tasks. ANNs comprise of two main elements; the neurons or processing elements (PEs) and the connections between the PEs which have weight values. Figure 2 shows a schematic diagram of a basic PE model (Haykin, 1999).

PEs are normally arranged in layers, where at least three layers of PEs (input, hidden and output layers) are required to construct an ANN which is capable of solving non-linear problems. A variant of the three-layered ANN architecture is the Multi-Layer Perceptron (MLP) neural network as shown in Figure 3 (Haykin, 1999). In the figure, $x, w$ and $y$ represent the inputs, weights and output values, respectively. In classification and non-linear function approximation, MLP is the most common and popular ANN ever used today (Ventari and Lampinen, 2000; Sterjovski et al., 2005).

ANNs are not programmed. Instead, the supervised type of ANNs such as the MLP, learn from given sets of input-output examples, establishing a mapping between inputs and outputs by updating their weight connections, to become 'intelligent' (Bishop, 1994). They 'learn' to predict the correct class for a given sets of inputs based on a suitable learning algorithm. This work investigated the most suitable learning algorithm for MLP in the quest for flow regime classification using ECT data of various flow patterns.

The presented work is concerned with flow regime classification without the need for imaging. The direct approach involves artificial neural networks (ANNs) which have been trained to classify various type of the flow regime based on the sets of ECT data. The following section describes procedures for preparing a set of simulated data and presents the ECT sensor model used in the investigation. Subsequently, the development of an 'intelligent' MLP in classifying flow regimes is explained. The results of MLP performances based on various employed learning algorithms are then presented and discussed.

\section{Development of the MLP Classification System}

Sensors for an ECT system are simple electrodes. Figure 4 shows the ECT sensor's parameter dimensions designed for this investigation. The inner pipe (R1), outer pipe (R2) and screen wall (R3) radii have been chosen to be $1,1.2$ and 1.4 units, respectively. The measurements of unit coordinate are used to facilitate generic mapping of parameter dimensions. The primary sensor $(\beta)$ and guard electrodes $(\alpha)$ have subtended angles of $22^{\circ}$ and $2.5^{\circ}$, respectively, making gaps of $8^{\circ}$ between each adjacent primary electrodes.

In this research, the ECT sensor model is chosen to have 12 electrodes since this is a common number of sensors used for ECT systems. With 12 electrodes, 66 distinct differences in capacitance between all possible pairs of electrodes can be obtained. This number is based on the equation below

$$
m=\frac{N(N-1)}{2}
$$

where $\mathrm{N}$ is the number of electrodes. It has to be noted that an ECT sensor creates the highest sensitivity around the edge of a sensing area which is nearer to the sensor electrodes, and the sensitivity tends to decrease towards the centre of the sensing area which is farther from the electrodes (Xie et al., 1992).

Figure 5 shows a schematic diagram of six flow regimes focused in this work. An ECT simulator (Spink, 1996) has been used to generate ECT data for different flow patterns of all investigated flow regimes based on the defined dimensions of the ECT sensor parameters. Every set of the ECT data corresponding to each flow pattern are normalized using,

$$
C_{i, j}=\frac{C_{i, j}^{m}-C_{i, j}^{l}}{C_{i, j}^{h}-C_{i, j}^{l}}
$$

where $C_{i, j}^{m}$ is the measured capacitance, $C_{i, j}^{l}$ is the capacitance when the pipe is full with the lower permittivity material, and $C_{i, j}^{h}$ is the capacitance when the pipe is full with the higher permittivity material. 
The numbers of flow patterns generated for each flow regime are given in Table 1. Empty and full pipe regimes have only one flow pattern each. This is because only one type of material (i.e. gas or oil) exists for both flow regimes and fully occupies the pipeline. Unlike the full or empty regimes, the stratified has many different patterns since it can be simulated using different oil heights as well as different tilted angle. The same with stratified regime, bubble flow patterns can be simulated for various bubble radii, as well as different bubble locations and hence, a rather large number of patterns for bubble flow. The annular and core flow patterns are just the opposite of each other where a larger number of these flow patterns can be simulated by varying their radii sizes.

Once all data have been generated and normalized, they are divided into training, validation and testing sets of data based on 8:1:1 ratio, respectively. Then, the process of MLP development commences where MLP is put into training process. Figure 6 illustrates the MLP training procedure, which has been accordingly executed using MATLAB ${ }^{\circledR}$.

Initially, the best transfer functions for the MLPs' hidden and output PEs are investigated by employing the training procedures. Since the output PEs should give either ' 1 ' or ' 0 ', the most suitable transfer function to be applied to them is the logarithmic sigmoid (log). As for the hidden PEs, either log or hyperbolic tangent (tanh) sigmoid transfer function can be applied. Hence, in the initial stage of the development, for a fixed number of hidden PEs, two investigations have been carried out, involving the combinations of log-log and tanh-log for the hidden and output PEs, respectively.

The next stage is to develop "intelligent" MLP classifiers for flow regime classification. In this case, three different learning algorithms; Levenberg-Marquardt (LM), Quasi-Newton (QN) and Resilient-Backpropagation (RP), have been investigated to determine the most suitable algorithm for the application. These algorithms are utilised because they are the commonly used algorithms for classification application. In doing so, for each learning algorithm, the number of hidden PEs for MLPs is increased (i.e. using the network growing approach), and their CCPs are recorded. The best algorithm is selected based on the highest CCP achieved by the MLP at a certain number of hidden PEs for that algorithm to ensure the network has learned the training process.

\section{Results and Discussion}

The CCPs for both investigations have been obtained as depicted in Table 2. The results show that the tanh-log combination of transfer functions gives higher CCP compared to log-log, although the difference is rather small.

Figure 7, 8 and 9 illustrate the results for CCP versus the number of hidden neurons for test dataset using LM, QN and RP, respectively. Clearly, from the plot in Figure 7, it can be seen that the LM algorithm is capable of training the MLP to obtain $100 \%$ CCP starting at 11 hidden PEs. This shows that LM algorithm has the ability to avoid local minima traps, a problem that always occurs in ANN training. Obviously, compared to other two training algorithms, the LM algorithm thus appears to be the best algorithm. This may be because LM algorithm has a robust minimum-searching strategy and the capability to jump out of the local minima (Bishop, 1994). Meanwhile, the highest CCP achieved for both QN and RP algorithms are 99.3\%. This shows that the MLP trained with the LM algorithm classifies the test data better than the MLP trained with the QN and RP algorithms.

Table 3 shows the CCP for each of the flow regimes based on the test dataset. The full and empty regimes give $0 \% \mathrm{CCP}$ because these flow patterns have been included only in the training data, and none in the test data since there is only one flow pattern for each regime. Among the flow regimes, stratified gives the highest CCP of about $43.75 \%$. This could be because stratified regime has the largest number of patterns in the training datasets (612 patterns) and hence, the MLP could classify stratified patterns the best. In addition, it is easier to identify a stratified pattern because the interface of differing permittivity (i.e. gas and oil) can be identified at the edge of the pipe, where the sensitivity of the sensing area is the highest.

The second highest CCP is $43.72 \%$, obtained from the bubble regime, which has the second largest number of patterns in the training dataset.

Although the core and annular regimes have the same number of patterns in the training dataset, their CCP differs due to the differing sensitivity effect they contribute to the ECT sensor. Based on the results, the MLP can identify the core flow patterns better than annular.

\section{Conclusion}

The work proposed a direct method for flow regime classification of gas-oil flows using MLP neural network as the classifier, based on simulated ECT data. In developing the MLP classifier for the task, the LM, QN and RP learning techniques have been investigated to determine the most suitable algorithm for the task of classifying six different flow regimes. The results demonstrated superiority of MLP when trained with the LM algorithm due to a $100 \%$ correct classification of unseen test data. Detailed analyses of each flow regime shows that the MLP could best identify stratified flow patterns. This could be because most of the training set consists of ECT data corresponding to this regime. In the future, it would be interesting to investigate the effect of simulated data replication on the MLP performance, particularly for ECT research where only limited number of patterns can be obtained for some classes such as with the 
empty and full regimes. Such investigation results could prove useful for other classification application that has bias (i.e. different) numbers of training sets for different classes.

\section{References}

Beck, M.S. and Williams, R.A. (1996). Process Tomography: A European innovation and its application. Flow Measurement and Instrumentation, 7, pp. 215-224.

Bishop, C.M. (1994). Neural Network and Their Application. Review of Scientific Instruments, 65(6), pp. $1803-1832$.

Gamio, J.C., Castro, J., Rivera, L., Alamila, J., Garcia-Nocetti, F. and Aguilar L. (2005). Visualisation of gas-oil two-phase flows in pressurised pipes using electrical capacitance tomography. Flow Measurement and Instrumentation, 16(2-3), 2005, pp 129- 134.

Haykin, S. (1999). Neural Networks: A Comprehensive Foundation. (1st ed.). Englewood Cliffs: Prentice Hall, (Chapter 1).

Marashdeh, Q., Warsito, W., Fan, L-S and Teixera F.L. (2006). A nonlinear image reconstruction technique for ECT using a combined neural network approach. Measurement Science and Technology, 17, pp. 2097-2103.

Mohamah-Saleh, J. and Hoyle, B.S. (2002). Determination of multi-component flow process parameters based on electrical capacitance tomography data using artificial neural networks. Measurement Science and Technology, 13, pp. 1815-1821.

Spink, D.M. (1996). Direct finite element solution for the capacitance, conductance or inductance, and force in linear electrostatic and magnetostatic problems. The International Journal for Computation and Mathematics in Electrical and Electronic Engineering, 15(3), pp. 70-84.

Sterjovski, Z., Dolan, A., Carpenter, K.R, Dunne, D.P. and Norrish, J. (2005). Artificial neural networks for modeling the mechanical properties of steels in various applications. Journal of Materials Processing Technology, 170, pp. 536-544.

Ventari, A. and Lampinen, J. (2000). Bayesian MLP neural networks for image analysis. Pattern Recognition Letters, 21 , pp. 1183-1181.

Xie, C.G., Huang, S.M., Hoyle, B.S., Thorn, R., Lenn, C., Snowden, D. and Beck, M.S. (1992). Electrical Capacitance Tomography for Flow Imaging. IEE Proceedings $-G$, 139(1), pp. 89-98.

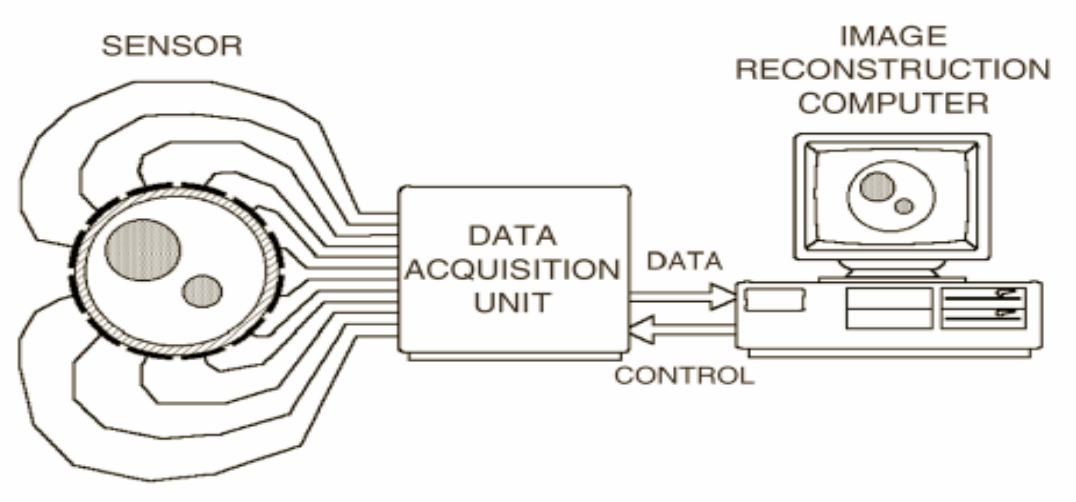

Figure 1. A basic ECT system 


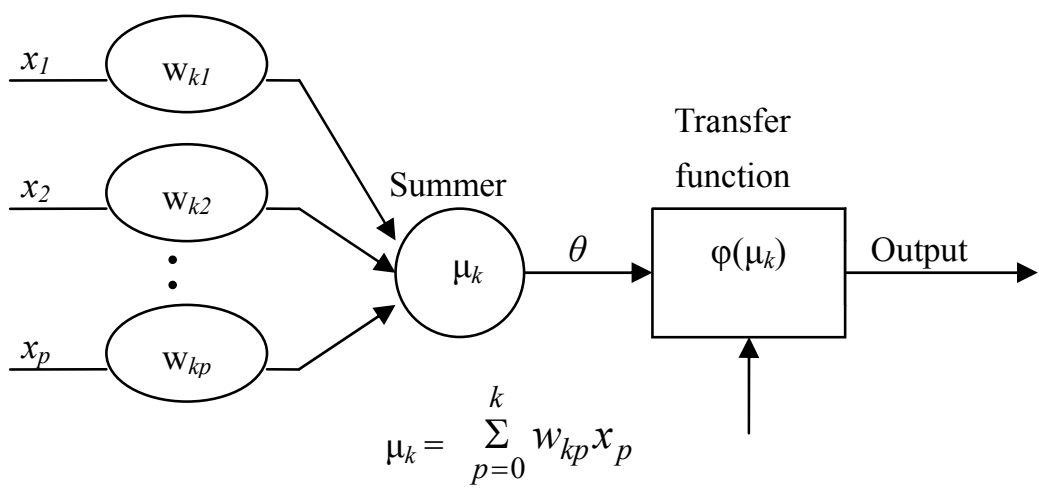

Threshold value

Figure 2. A basic PE model

Input weight Output weight

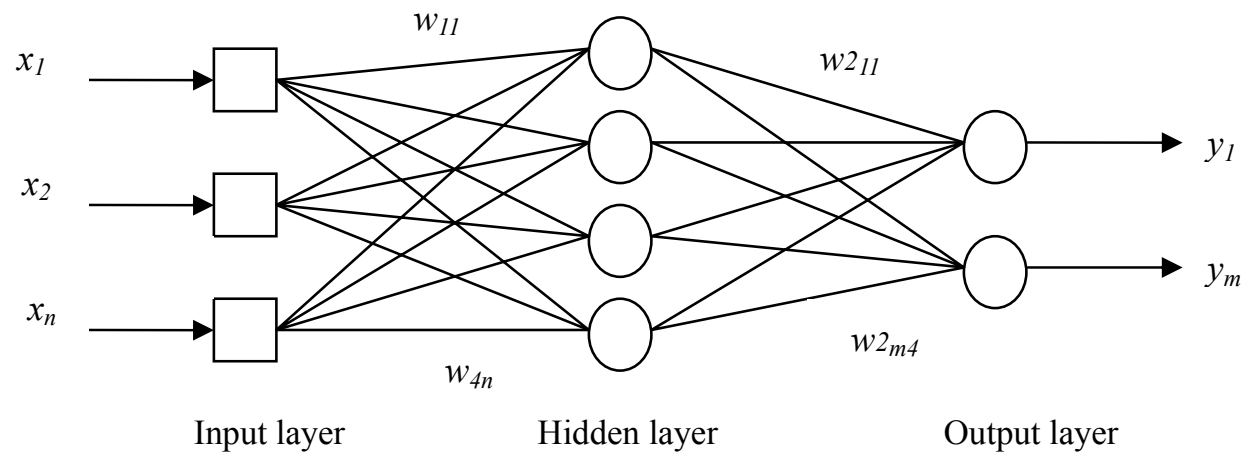

Figure 3. A schematic diagram of a Multi-Layer Perceptron (MLP) neural network

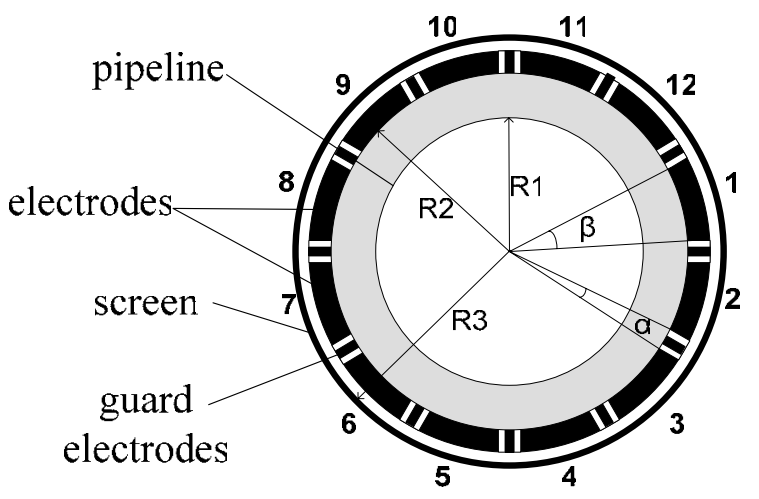

Figure 4. ECT sensor model 


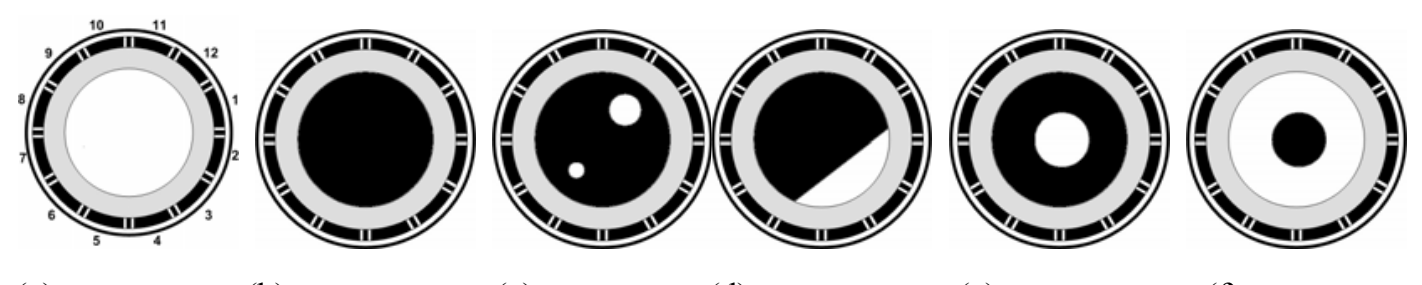

(a)

(b)

(c)

(d)

(e)

(f)

Figure 5. Schematic diagram of the flow regimes to be classified:

(a) empty (b) full (c) bubblen (d) stratified (e) annular (f) core

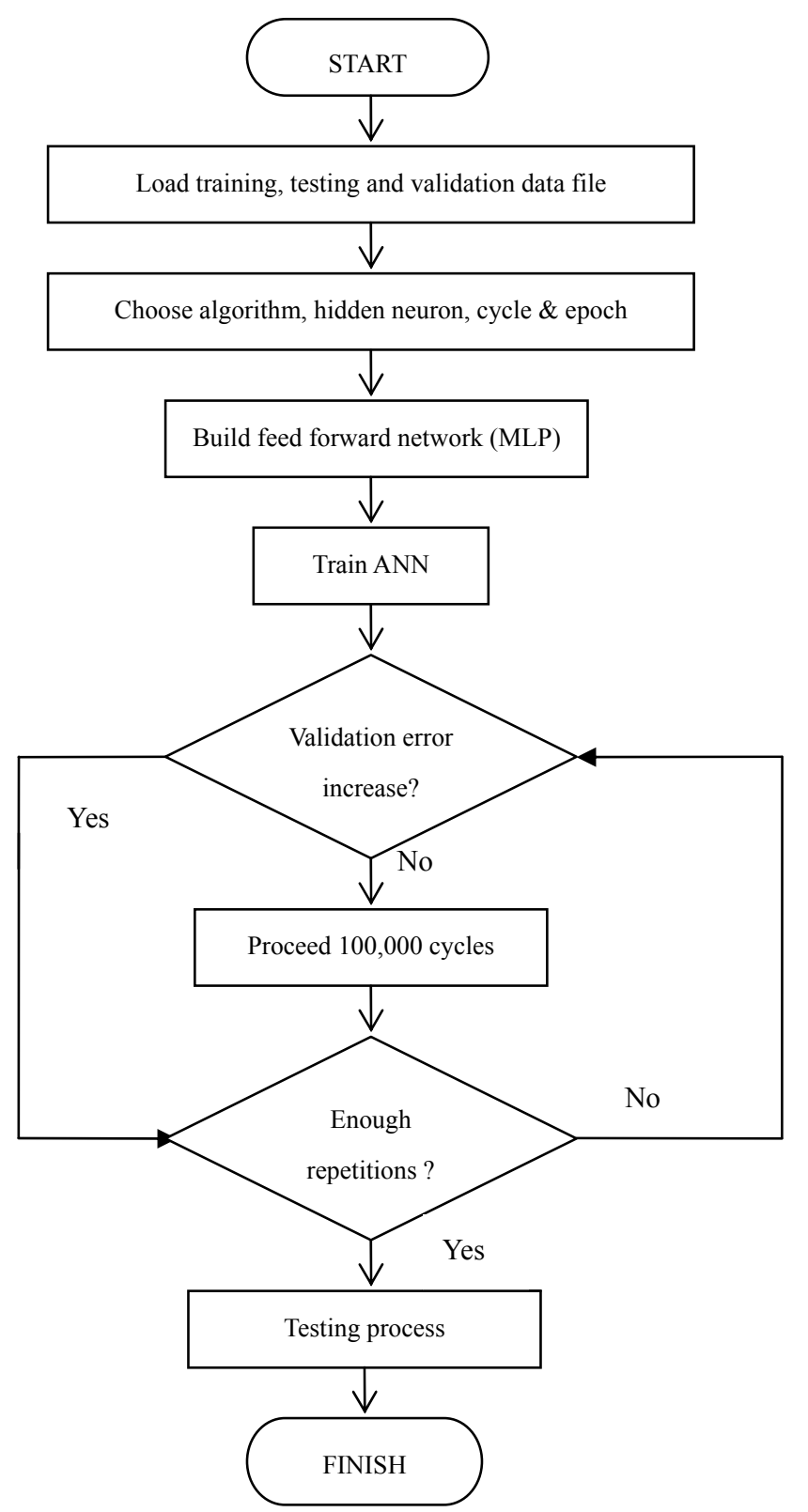

Figure 6. Flowchart of MLP training process 


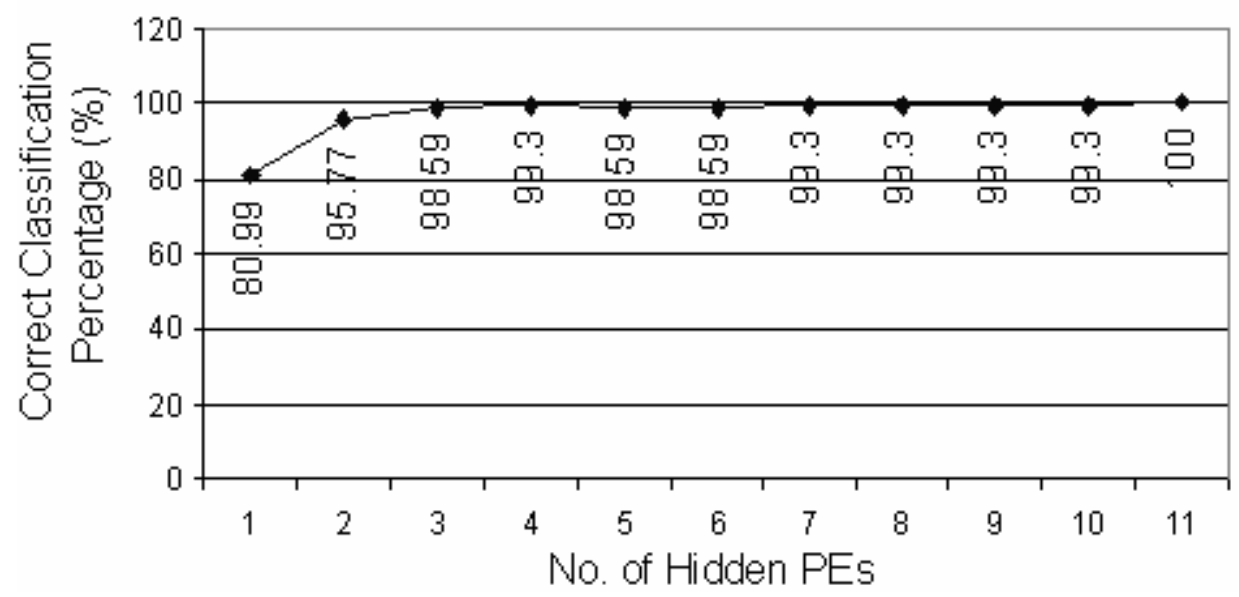

Figure 7. CCP for test dataset based on LM algorithm at various numbers of hidden PEs

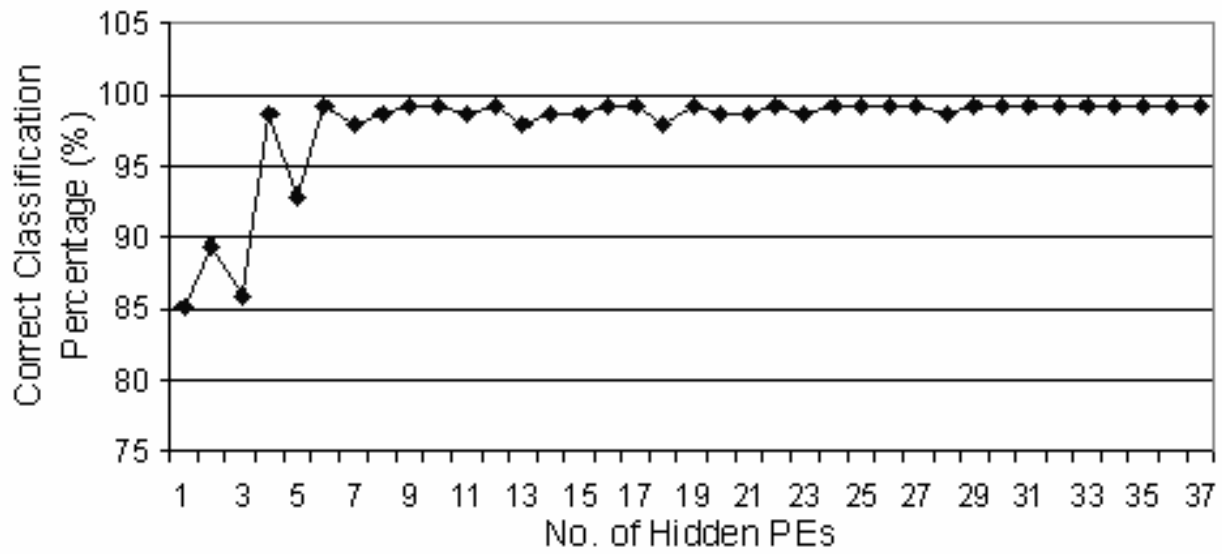

Figure 8. CCP for test dataset based on QN algorithm at various numbers of hidden PEs

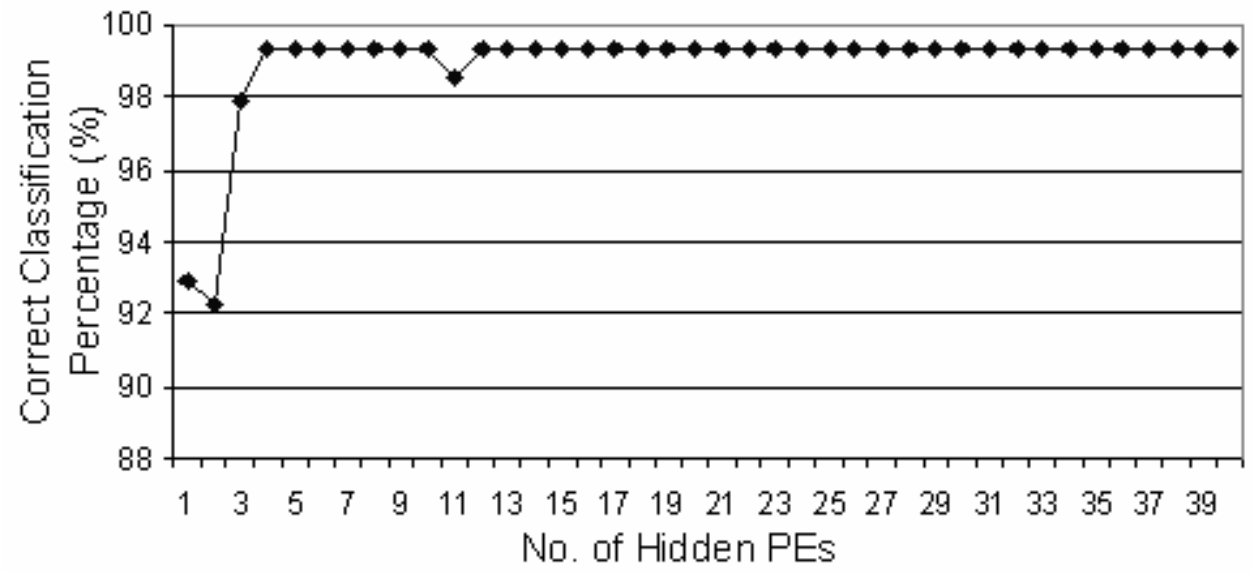

Figure 9. CCP for test dataset based on RP algorithm at various numbers of hidden PEs 
Table 1. The numbers of for each flow regime

\begin{tabular}{|c|c|}
\hline Flow Regime & No. flow patterns \\
\hline Full & 1 \\
\hline Stratified & 612 \\
\hline Bubble & 520 \\
\hline Core & 99 \\
\hline Annular & 99 \\
\hline Empty & 1 \\
\hline Total & 1332 \\
\hline
\end{tabular}

Table 2. Performance of MLP for different combinations of transfer function

\begin{tabular}{|c|c|}
\hline Combination of transfer function & CCP $(\%)$ \\
\hline Log-Log & 97.2 \\
\hline Tanh-Log & 98.6 \\
\hline
\end{tabular}

Table 3. CPP of each flow regime for the best MLP based on test datasets

\begin{tabular}{|c|c|c|c|c|c|}
\hline \multicolumn{7}{|c|}{ CCP $(\%)$} \\
\hline Full & Stratified & Bubble & Core & Annular & Empty \\
\hline 0 & 43.75 & 43.72 & 6.79 & 5.74 & 0 \\
\hline
\end{tabular}

